Timing Channels in Cryptography 
Chester Rebeiro - Debdeep Mukhopadhyay Sarani Bhattacharya

\section{Timing Channels in Cryptography}

A Micro-Architectural Perspective 
Chester Rebeiro

Columbia University

New York

New York

USA

Debdeep Mukhopadhyay

Indian Institute of Technology

Kharagpur

West Bengal

India
Sarani Bhattacharya

Department of Computer Science and Engin

IIT Kharagpur

Kharagpur

India

ISBN 978-3-319-12369-1

ISBN 978-3-319-12370-7 (eBook)

DOI 10.1007/978-3-319-12370-7

Library of Congress Control Number: 2014954350

Springer Cham Heidelberg New York Dordrecht London

(C) Springer International Publishing Switzerland 2015

This work is subject to copyright. All rights are reserved by the Publisher, whether the whole or part of the material is concerned, specifically the rights of translation, reprinting, reuse of illustrations, recitation, broadcasting, reproduction on microfilms or in any other physical way, and transmission or information storage and retrieval, electronic adaptation, computer software, or by similar or dissimilar methodology now known or hereafter developed.

The use of general descriptive names, registered names, trademarks, service marks, etc. in this publication does not imply, even in the absence of a specific statement, that such names are exempt from the relevant protective laws and regulations and therefore free for general use.

The publisher, the authors and the editors are safe to assume that the advice and information in this book are believed to be true and accurate at the date of publication. Neither the publisher nor the authors or the editors give a warranty, express or implied, with respect to the material contained herein or for any errors or omissions that may have been made.

Printed on acid-free paper

Springer is part of Springer Science+Business Media (www.springer.com) 


\section{Foreword}

With the publication of the Data Encryption Standard DES in 1977 and the invention of public key cryptography in the 1980s, cryptography has moved into civil applications. This was pushed by the electronic revolution, which needs cryptography to create an electronic equivalent of the real world into the digital world. Cryptography is used to provide confidentiality, digital signatures, anonymity, payments, electronic transactions, elections, and many more.

Cryptography and cryptographic protocols by themselves do not provide security: they need a digital platform that executes the algorithms and protocols. In the early days, these platforms were computers and servers sitting in well protected computer rooms and offices. The main concern when implementing the algorithms and protocols was efficiency, as cryptographic algorithms are typically very computationally demanding. The attacker model assumed that the computer platforms themselves were well protected and that only the result of the encryption, the ciphertext, has to travel over insecure communication channels, such as cables or satellite communication or tapes that are transported between bank offices and the bank headquarters.

However, as the electronic revolution expanded, electronics became cheaper, more widespread, and integrated into day to day applications. Examples are pay TV systems, banking cards, identity cards, or access control systems. This omnipresence of electronic gadgets changes the attacker model! Now, the implementation becomes the weak link in the chain: the attacker will attack while the electronic device is performing cryptographic operations using the secret key operating on possible sensitive data. During calculations, the devices leak information. These are the so-called side channel attacks. The attacker will observe the device while it performs its calculations. From timing or power consumption variations, or from electromagnetic radiations, the attacker is able to guess which operations and which sensitive data is being handled.

The contributions of this book are situated in this context. One highly important source of side channel information leakage is the timing variations of calculations. Cryptographic algorithms and protocols are written in high level languages and compiled onto sophisticated digital platforms. Both the software stack and the hardware platform include a large variety of optimizations to improve performance. Memory 
accesses and branches are expensive in execution time, therefore processors include caches and branch predictors. Unfortunately, these optimizations result in timing variations when executing a program. Caches are considered one of the most important sources of side channel information leakage. This book therefore gives a comprehensive overview and an in depth analysis of many flavors of attacks using caches and branch predictors. It ends with a set of countermeasures to address these timing attacks at different abstraction layers.

This book is a fine read for anyone interested in the many possibilities of cache attacks. A software engineer should not write any line of a cryptographic routine without knowing how powerful timing attacks are. This book gives a clear insight on how these attacks work and how they can be mitigated. Enjoy reading!

Leuven, Belgium

Ingrid Verbauwhede

December 2014 


\section{Preface}

Cryptography plays a vital role in securing e-business and e-commerce transactions. The algorithms used have been rigorously analyzed and tested for their ability to conceal information. However, these algorithms are needed to be realized in systems that are used in a variety of applications. As the saying says, there's many a slip 'twixt the cup and the lip, these implementations may leak sensitive information via unintended timing channels. Over the past 20 years, several attacks have been developed that use these side-channels to reveal secrets from ciphers. These attacks, known as timing attacks, are powerful enough to break a mathematically robust cipher in few minutes on standard computing platforms.

Starting from its inception to the present day, timing attacks and the underneath statistics have evolved. Enhancements made to computer architecture over the years have also influenced timing channels. To develop a system secure against these threatening channels, one needs to be abreast of the interplay between cryptographic algorithms and computer architecture. Here is where this book steps in. It brings on a single platform aspects of computer architecture and cryptography that are essential to understand how timing attacks work and why they work. It describes the attacks and analyzes the relationship between the cipher implementation, system microarchitecture, and the attack threat. Various timing channels arising due to cache memories and branch prediction units are presented. The book would help engineers and researchers understand timing attacks and thereafter develop platforms that can tolerate these attacks.

The following topics are covered.

- Modern Cryptography. Attackers make use of the cipher's structure to extract the secret key from execution time. The attacks are made even more powerful when ideas from classical cryptanalysis are used with the timing information. The book therefore begins with a review of a variety of modern ciphers ranging from symmetric algorithms like the Advanced Encryption Standard (AES) to asymmetric algorithms like the Rivest-Shamir-Adleman (RSA), and some popular classical cryptanalysis techniques. 
- Superscalar Processor Architectures. To address how timing channels arise in a system, the book provides a background of the internal micro-architecture of processors. An introduction to modern superscalar architectures has been provided, with an emphasis on the components in them that affect the execution time of ciphers.

- Time-Driven Cache Attacks on Block Ciphers. Memory load instructions that cause a cache miss takes considerably longer than those that result in a cache hit. Attackers utilize this difference to build a variety of attacks on block ciphers. A detailed discussion on how these attacks are implemented is presented, with emphasis on various accurate time measurement techniques and analysis methods to observe the cache hit miss phenomenon.

- Advanced Time-Driven Cache Attacks on Block Ciphers. Combination of classical cryptanalysis with side-channel attacks paves way to powerful attack methodologies. A combination of classical differential cryptanalysis with cache timing attacks has been discussed in the book to describe this threat.

- Formal Analysis of Time-Driven Cache Attacks. Developing suitable metrics for comparison of secured implementations and using them to guide the design flow is an important aspect in security engineering. In this pursuit, the book provides formal modeling of time-driven cache attacks on block ciphers and suggests metrics for evaluation and comparison of such implementations. A discourse on how micro-architectural features, such as pipelining and out-of-order execution, affect cache attacks is presented. These guidelines form a framework for developing ideal implementations of the block ciphers with respect to time-driven cache attacks.

- Profiled Time-Driven Cache Attacks on Block Ciphers. There are various types of cache attacks, of which profiled cache attacks are arguably the most powerful. The book provides a detailed description of such profiled attacks, which rely on building timing profiles in a learning phase before mounting the attack. The book also provides insights into developing metrics for estimating the information leakage and relates the leakage to micro-architectural features in modern computers, such as hardware prefetchers.

- Access-Driven Cache Attacks on Block Ciphers. Cache attacks come in different flavors, one of them, called access attacks, relies on a spy program running along with the encryption program. The spy uses timing measurements to determine memory access patterns made by the encryption. By monopolizing the OS scheduler, the spy program can accurately determine every memory access made, thereby leading to powerful attacks. The book provides a comprehensive overview on the topic to understand the theory and develop the procedure of such access attacks.

- Branch Prediction Attacks. Branch prediction units are artifacts in computer architectures to reduce performance penalties due to branch instructions. They predict whether a branch instruction will be taken or not taken, thereby reducing processor stalls. Athough this can boost performance of an application significantly, it can also lead to timing channels that are catastrophic. Attackers have used these channels to break mathematically strong asymmetric key ciphers such 
as the RSA. Several of the attack strategies developed are discussed in detail in the book.

- Countermeasures. To circumvent timing attacks, a variety of countermeasures have been proposed at various levels in the system: from the application level and architectural level to the Operating System. The book tries to provide an overview on these countermeasures, how they are applied, their effectiveness, and the consequent overheads involved.

IIT Kharagpur, India

Chester Rebeiro

November 2014 Debdeep Mukhopadhyay

Sarani Bhattacharya 


\section{Acknowledgements}

This preface would be incomplete without a mention of the people who made this effort possible. The authors would like to express their sincere gratitude to the Computer Science and Engineering Department of IIT Kharagpur, which provides an ideal environment for research on information security. The authors would like to thank all their colleagues, students, collaborators, and funding agencies without whose support this work would not be possible. Special thanks to Abhijit Das, Partha Pratim Chakrabarty, Rajat Subhra Chakraborty, Dipanwita Roy Chowdhury, Phuong Ha Nguyen, and Indranil Sengupta from IIT Kharagpur, and Junko Takahashi (NTT Labs), Toshinori Fukunaga (NTT Labs), and Sobha PM (CDAC Bangalore) for their help and support during the project.

Chester Rebeiro would like to thank his family members especially his parents, wife Sharon, and son Tristan for their love and prayers.

Debdeep Mukhopadhyay would like to thank in particular all his family members and friends. He would like to make a special mention of his parents, wife, and brother for their bonding and wishes. He would like to dedicate this book to his daughter Debanti, who has indeed made his life more interesting.

Sarani Bhattacharya would like to thank her parents and friends for their continuous support and encouragement. She would also like to specially thank her mentor Prof. Debdeep Mukhopadhyay for his invaluable guidance and Dr. Chester Rebeiro for his advice and suggestions. 


\section{Contents}

1 An Introduction to Timing Attacks $\ldots \ldots \ldots \ldots \ldots \ldots \ldots \ldots \ldots \ldots \ldots$

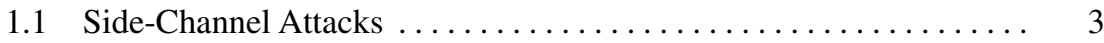

1.1.1 Side-Channel Attack Requirements $\ldots \ldots \ldots \ldots \ldots \ldots \ldots$

1.1.2 The Attacker's Success . . . . . . . . . . . . . . . ..... 5

1.1.3 Side-Channel Attack Suppression ................ 5

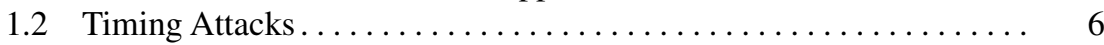

1.2.1 Kocher's Timing Attack $\ldots \ldots \ldots \ldots \ldots \ldots \ldots \ldots \ldots \ldots$

1.2.2 Taxonomy of Timing Attacks $\ldots \ldots \ldots \ldots \ldots \ldots$

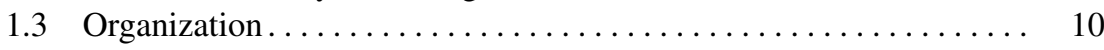

Reference ................................. 11

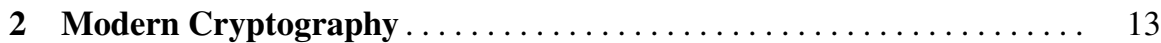

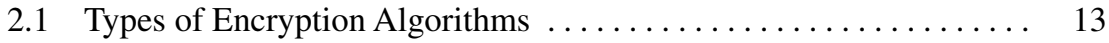

2.2 Block Ciphers: An Important Family of Symmetric-Key Ciphers ... 15

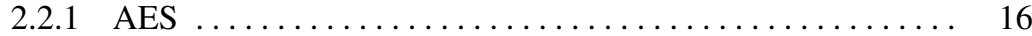

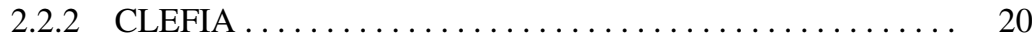

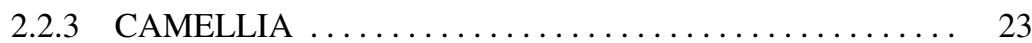

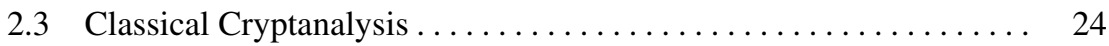

2.3.1 Classical Cryptanalysis of Block Ciphers ........... 25

2.3.2 The Idea of Differential in Block Ciphers ........... 25

2.4 Asymmetric-Key Ciphers . . . . . . . . . . . . . . . . . . . 29

2.5 RSA: An Asymmetric-Key Algorithm .................... 29

2.5.1 Square and Multiply Algorithm to Perform Exponentiation . 30

2.6 Confinement Problem and Covert Channels ................ 31

2.7 Formal Analysis of Side-Channel Attacks $\ldots \ldots \ldots \ldots \ldots \ldots \ldots . \ldots \ldots$

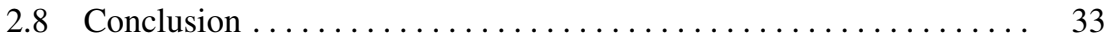

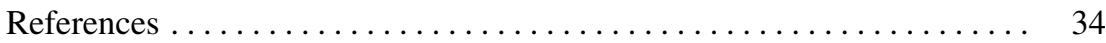

3 Superscalar Processors, Cache Memories, and Branch Predictors . . 37

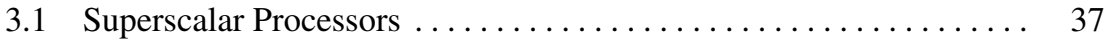

3.2 Memory Hierarchy and Cache Memory ... . . . . . . . . . . . 39 
3.2.1 Organization of Cache Memory $\ldots \ldots \ldots \ldots \ldots \ldots \ldots \ldots 40$

3.2.2 Improving Cache Performance for Superscalar Processors . . 43

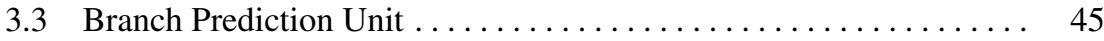

3.3.1 Static Branch Prediction................... 47

3.3.2 Dynamic Branch Prediction Schemes.............. 47

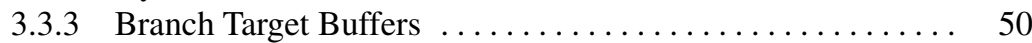

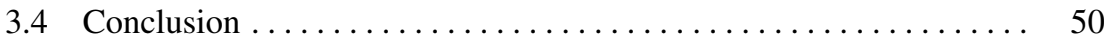

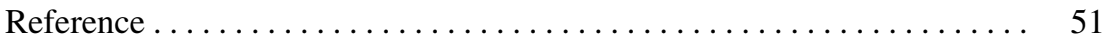

4 Time-Driven Cache Attacks $\ldots \ldots \ldots \ldots \ldots \ldots \ldots \ldots \ldots \ldots \ldots \ldots$

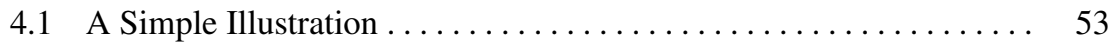

4.1.1 Relation Between Size and Bits Revealed ............ 55

4.1.2 Relation Between Alignment of Tables and Bits Revealed... 56

4.1.3 Initial State of Cache Memory $\ldots \ldots \ldots \ldots \ldots \ldots \ldots \ldots . \ldots 6$

4.2 Collisions from Execution Time . . . . . . . . . . . . . 57

4.2.1 Clocks Using Hardware Time Stamp Counters . . . . . . . . . 57

4.2.2 Clocks with Virtual Time-Stamp Counters ............ 59

4.2.3 Distinguishing Cache Hit and Miss Events Using Time . . . . 60

4.3 Timing Attacks on Block Ciphers Based on Internal Collisions . . . . . 63

4.3.1 Max, Min, or Max Deviation .................. 65

4.4 Time-Driven Attack Based on Induced Cache Miss . . . . . . . . . 66

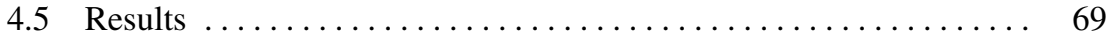

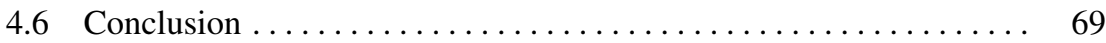

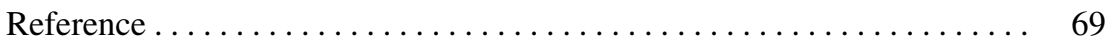

5 Advanced Time-Driven Cache Attacks on Block Ciphers . . . . . . . . 71

5.1 Second Round Attack on AES ..................... 71

5.2 Differential Cache Attacks on Feistel Ciphers . . . . . . . . . . . 72

5.3 Differential Cache Attack on CLEFIA ................ 75

5.3.1 Differential Properties of CLEFIA's $F$ Functions . . . . . . . 75

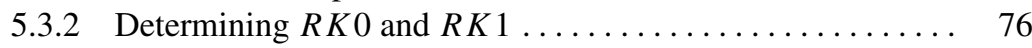

5.3.3 Determining $W K 0 \oplus R K 2$ and $W K 1 \oplus R K 3 \ldots \ldots \ldots . \ldots 77$

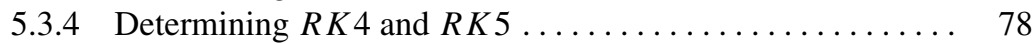

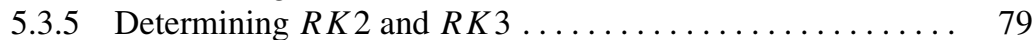

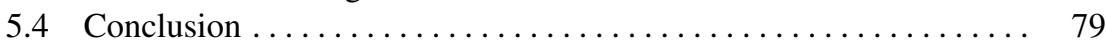

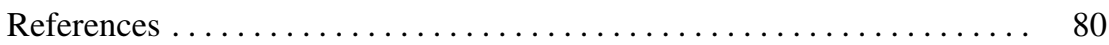

6 A Formal Analysis of Time-Driven Cache Attacks $\ldots \ldots \ldots \ldots \ldots$. . . . 81

6.1 Memory Access Model for a Block Cipher ............. 81

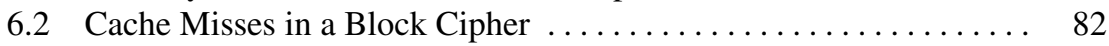

6.3 Average Execution Time of a Block Cipher ............. 85

6.3.1 Estimating the Difference of Means . . . . . . . . . . . 87

$6.4 \mathrm{DOM}$ as a Security Metric $\ldots \ldots \ldots \ldots \ldots \ldots \ldots \ldots \ldots$

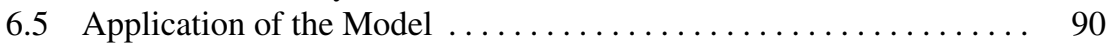


6.5.1 Comparing Cipher Implementations . . . . . . . . . . . . 91

6.5.2 Choosing the Right Implementation . . . . . . . . . . . . 92

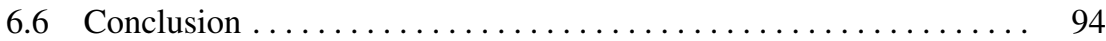

7 Profiled Time-Driven Cache Attacks on Block Ciphers $\ldots \ldots \ldots \ldots \ldots 95$

7.1 Bernstein's Cache Timing Attack $\ldots \ldots \ldots \ldots \ldots \ldots \ldots \ldots \ldots$

7.1.1 Building a Timing Profile $\ldots \ldots \ldots \ldots \ldots \ldots \ldots \ldots$

7.1.2 Extracting Keys from Timing Profiles ............ 97

7.2 Causes of Information Leakage . . . . . . . . . . . . . . . . 98

7.3 Quantifying Information Leakage in a Timing Profile . . . . . . . . . 103

7.4 Information Leakage due to Hardware Prefetching . . . . . . . . . . . 104

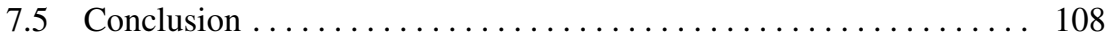

References .................................. 108

8 Access-Driven Cache Attacks on Block Ciphers . . . . . . . . . . . . . . . . 109

8.1 Access-Driven Attacks on Block Ciphers . . . . . . . . . . . . . . . . . 109

8.1.1 Second Round Access-Driven Attack on AES . . . . . . . . 112

8.1.2 A Last Round Access-Driven Attack on AES . . . . . . . . . . 112

8.2 Asynchronous Access-Driven Attacks . . . . . . . . . . . . . . . . 113

8.3 Secretly Monopolizing the CPU scheduler . . . . . . . . . . . . . . . 114

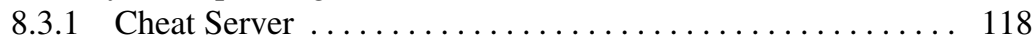

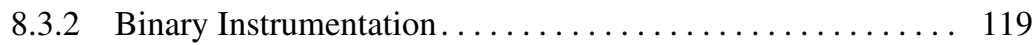

8.4 Fine Grained Access-Driven Attack on AES . . . . . . . . . . . . . . . . 119

8.5 Attack Procedure . . . . . . . . . . . . . . . . . . . . . . . 120

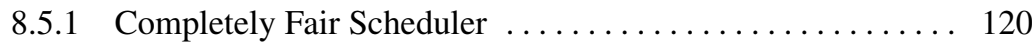

8.5.2 Denial of Service Exploiting Completely Fair Scheduler . . . 121

8.5.3 Using Timing as Side Channel for Cache Access Attack . . . 122

8.5 .4 Denoising by Neural Network ................. 123

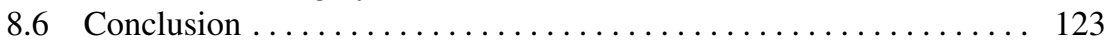

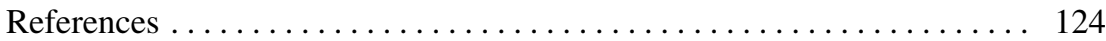

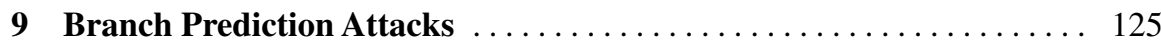

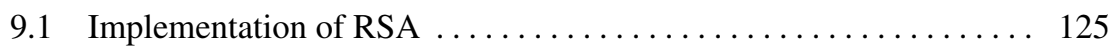

9.1.1 Square and Multiply Exponentiation Algorithm . . . . . . . . 125

9.1.2 Balanced Montgomery Powering Ladder Implementation . . 126

9.1.3 Montgomery Multiplication $\ldots \ldots \ldots \ldots \ldots \ldots \ldots \ldots \ldots$

9.2 Timing Branch Mispredictions . . . . . . . . . . . . . . . 127

9.3 Attacking the Square and Multiply Exponentiation Algorithm. . . . . . 130

9.4 Asynchronous Attack on the Square and Multiply Algorithm . . . . . 134

9.5 Synchronous Attack on the Square and Multiply Algorithm . . . . . 136

9.6 Trace Driven Attack Targeting the BTB . . . . . . . . . . . . . 136

9.7 Conclusion .............................. 137

Reference..................................... 137 
10 Countermeasures for Timing Attacks . . . . . . . . . . . . . . . . 139

10.1 Application Level Countermeasures . . . . . . . . . . . . . . . 139

10.1.1 Countermeasures Involving Look-Up Tables . . . . . . . . 140

10.1.2 Data-Oblivious Memory Access Pattern . . . . . . . . . . . 142

10.1.3 Constant and Random Time Implementations . . . . . . . . . 142

10.2 Countermeasures Applied in the Hardware . . . . . . . . . . . . . 143

10.2.1 Noncached Memory Accesses . . . . . . . . . . . . . . . . . . . 143

10.2.2 Specialized Cache Designs $\ldots \ldots \ldots \ldots \ldots \ldots \ldots \ldots \ldots \ldots 143$

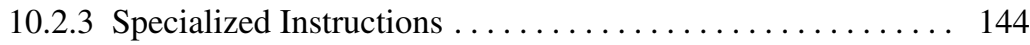

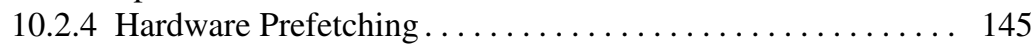

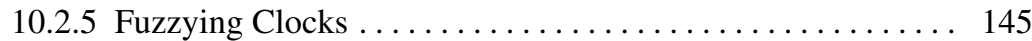

10.3 Countermeasures in the Operating System . . . . . . . . . . . 146

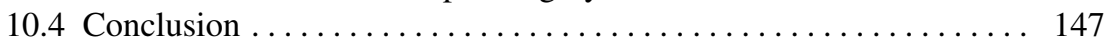

References ..................................... 147

Appendix A: CPUs Used for Experiments $\ldots \ldots \ldots \ldots \ldots \ldots \ldots \ldots \ldots \ldots$ 


\section{List of Abbreviations}

$\begin{array}{ll}\text { AES } & \text { Advanced Encryption Standard } \\ \text { BEEA } & \text { Binary Extend Euclidean Algorithm } \\ \text { BPU } & \text { Branch Prediction Unit } \\ \text { BTB } & \text { Branch Target Buffer } \\ \text { CFS } & \text { Completely Fair Scheduler } \\ \text { CCA } & \text { Chosen Ciphertext Attack } \\ \text { CPA } & \text { Chosen Plaintext Attack } \\ \text { CPU } & \text { Central Processing Unit } \\ \text { CMOS } & \text { Complementary Metal Oxide Semiconductor } \\ \text { CRT } & \text { Chinese Remainder Theorem } \\ \text { DOM } & \text { Difference of Means } \\ \text { DRAM } & \text { Dynamic Random Access Memory } \\ \text { GCD } & \text { Greatest Common Divisor } \\ \text { GF } & \text { Galois Field } \\ \text { GFN } & \text { Generalized Feistel Networks } \\ \text { IPC } & \text { Inter Process Communication } \\ \text { ISA } & \text { Instruction Set Architecture } \\ \text { L1 } & \text { Level 1 } \\ \text { L2 } & \text { Level 2 } \\ \text { L3 } & \text { Level 3 } \\ \text { MM } & \text { Montgomery Multiplication } \\ \text { NIST } & \text { National Institute Standards and Technology } \\ \text { NSS } & \text { Network Security Services } \\ \text { NTT } & \text { Nippon Telegraph and Telephone Corporation } \\ \text { PC } & \text { Program Counter } \\ \text { RAM } & \text { Random Access Memory } \\ \text { RSA } & \text { Rivest Shamir Adleman } \\ \text { SCA } & \text { Side Channel Attacks } \\ \text { SMT } & \text { Symmetrical Multithreading } \\ \text { SNR } & \text { Signal to Noise Ratio } \\ \text { SPN } & \text { Substitution Permutation Network } \\ \text { SRAM } & \text { Static Random Access Memory } \\ \text { TSC } & \text { Time Stamp Counter } \\ \text { VTSC } & \text { Virtual Time Stamp Counter } \\ & \end{array}$

